\title{
Impact of shock wave on a body moving at Supersonic speed
}

\author{
${ }^{1}$ Neeraj Jasher, ${ }^{2}$ Nirav Patel $\&{ }^{3}$ Blesson Prakash \\ ${ }^{1,2,3}$ Aerospace Engineering, Indian Institute of Aeronautical Engineering, Dehradun, India
}

Abstract: Determining how much force will an aircraft or any forward moving body have to face when it travels at a velocity of more than the velocity of sound. The core of this research is to find out how much pressure air can exert after the formation of the shock wave, subsequently calculating force.

Keywords

1. Drag: It is the air resistance caused due to the forward motion of any object such as aircrafts.

2. Shock wave: It is the thin layer of air caused when any object (aircraft in our case) exceeds the speed of sound $(330 \mathrm{~m} / \mathrm{s}$ at sea level). Due to shock wave drag increases rapidly.

3. Mach number: It is the ratio of the velocity of any object with respect to speed of sound.

$$
M=\frac{V}{\sqrt{\gamma R T}}
$$

\section{Introduction:}

The following figure 1 is the nose section of an aircraft and the position of the shock wave, the distance of the shock from the nose section of the aircraft is derived below:

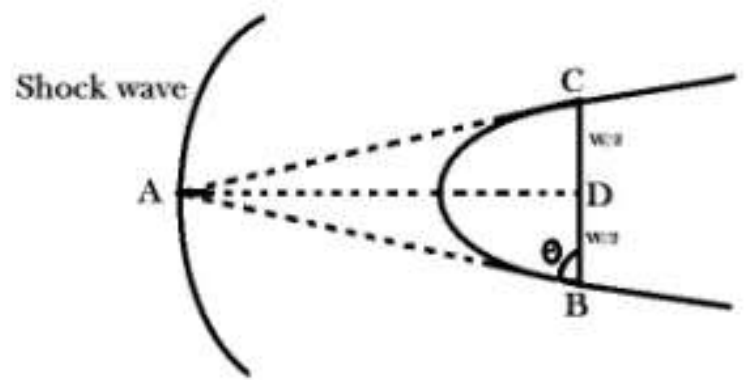

Figure 1 Magnified image of the formation of shock ahead of the nose of the aircraft

$$
\begin{aligned}
& =>\tan \theta=\frac{A D}{\frac{w}{2}} \\
& =>A D=\frac{w}{2} \tan \theta
\end{aligned}
$$

Hence, this is the distance where the shock wave is formed. $\frac{w}{2} \tan \theta$ Units away from the effective base area of the radom.

Now considering the effects due to the shock wave:

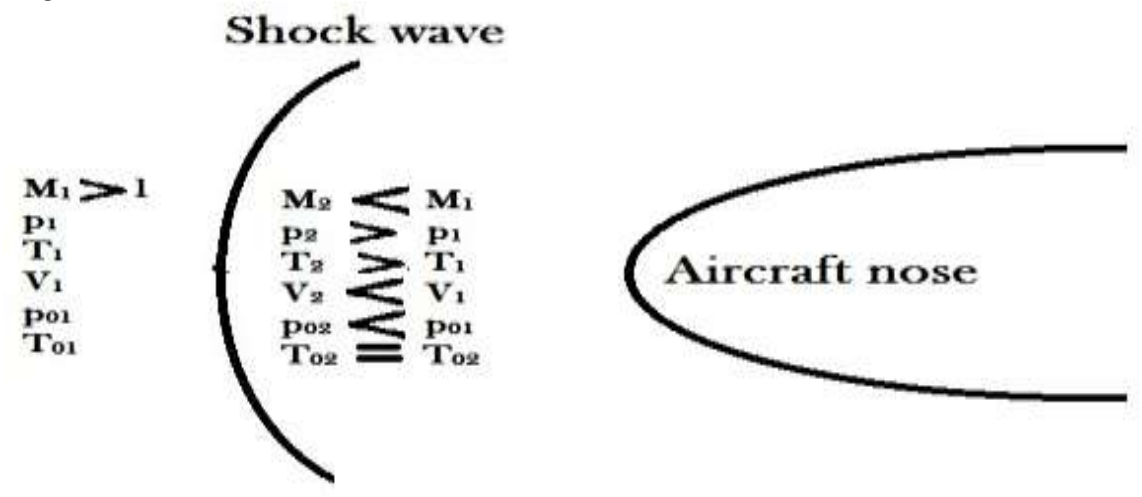

Figure 2 effects of shock wave 
First let's consider the isentropic flow relation after the shock wave:

$=>C_{p} T_{2}+\frac{V_{2}^{2}}{2}=C_{p} T_{\mathbf{m} 2}$

$=>1+\frac{V_{2}^{2}}{2 C_{p} T_{2}}=\frac{T_{\mathbf{m}}}{T_{2}}$

and since $c_{p}=\frac{\gamma R}{(\gamma-1)}$

$=>\frac{T_{\mathbf{m} 2}}{T_{2}}=1+\frac{V_{2}^{2}}{2 \gamma R T_{2}}(\gamma-1)$

since, $\left[\frac{T_{\mathbf{m} 2}}{T_{2}}\right]^{\frac{\gamma}{\gamma-1}}=\frac{P_{\mathbf{m} 2}}{P_{2}}$

$=>\frac{P_{\mathbf{n} 2}}{P_{2}}=\left[1+\frac{(\gamma-1)}{2} M_{2}^{2}\right]^{\frac{\gamma}{\gamma-1}}$

Now let's derive one more equation from Momentum equation:

$P_{1}+\rho V_{1}^{2}=P_{2}+\rho V_{2}^{2}$

$P_{1}\left(1+\frac{\rho_{1}}{P_{1}} V_{1}^{2}\right)=P_{2}\left(1+\frac{\rho_{2}}{P_{2}} V_{2}^{2}\right)$

$P_{1}\left(1+\frac{1}{R T} V_{1}^{2}\right)=P_{2}\left(1+\frac{1}{R T} V_{2}^{2}\right)$

$P_{1}\left(1+\frac{\gamma}{\gamma R T_{1}} V_{1}^{2}\right)=P_{2}\left(1+\frac{\gamma}{\gamma R T_{2}} V_{2}^{2}\right)$

$P_{1}\left(1+\frac{\gamma}{a_{1}^{2}} V_{1}^{2}\right)=P_{2}\left(1+\frac{\gamma}{a_{2}^{2}} V_{2}^{2}\right)$

Where ' $\mathrm{a}$ ' is the speed of sound $a=\sqrt{\gamma R T}$

$P_{1}\left(1+\gamma M_{1}^{2}\right)=P_{2}\left(1+\gamma M_{2}^{2}\right)$

And hence, we get the following result:

$=>\frac{P_{2}}{P_{1}}=\frac{1+\gamma M_{1}^{2}}{1+\gamma M_{2}^{2}} \ldots$

Now here let's solve for $M_{2}^{2}$ :

By the definition of critical Mach number:

$M^{* 2}=\frac{(\gamma+1) M^{2}}{2+M^{2}(\gamma-1)}$

$M_{1}^{* 2}=\frac{(\gamma+1) M_{1}^{2}}{2+(\gamma-1) M_{1}^{2}}$

$M_{2}^{* 2}=\frac{(\gamma+1) M_{2}^{2}}{2+(\gamma-1) M_{2}^{2}}$

$M_{2}^{\star 2} M_{1}^{\star 2}=1$

$\frac{(\gamma+1) M_{2}^{2}}{2+(\gamma-1) M_{2}^{2}} X \frac{(\gamma+1) M_{1}^{2}}{2+(\gamma-1) M_{1}^{2}}=1$ 
$(\gamma+1)^{2} M_{1}^{2} M_{2}^{2}=4+(2 \gamma-2) M_{2}^{2}+(2 \gamma-2) M_{1}^{2}+(\gamma-1)^{2} M_{1}^{2} M_{2}^{2}$

2y $M_{1}^{2} M_{2}^{2}=2+(\gamma-1) M_{2}^{2}+(\gamma-1) M_{1}^{2}$

$2 \gamma M_{1}^{2} M_{2}^{2}-(\gamma-1) M_{2}^{2}=2+(\gamma-1) M_{1}^{2}$

$M_{2}^{2}=\frac{2+(\gamma-1) M_{1}^{2}}{2 \gamma M_{1}^{2}-(\gamma-1)}=\frac{1+\frac{(\gamma-1)}{2} M_{1}^{2}}{\gamma M_{1}^{2}-\frac{(\gamma-1)}{2}} \ldots \ldots$

Now applying equation 3 in equation 2, we get:

$\frac{P_{2}}{P_{1}}=\frac{1+\gamma M_{1}^{2}}{1+\gamma\left(\frac{1+\frac{(\gamma-1)}{2} M_{1}^{2}}{\gamma M_{1}^{2}-\frac{(\gamma-1)}{2}}\right)}$

$\frac{P_{2}}{P_{1}}=\frac{\left(1+\gamma M_{1}^{2}\right)\left(\gamma M_{1}^{2}-\frac{(\gamma-1)}{2}\right)}{\gamma M_{1}^{2}-\frac{(\gamma-1)}{2}+\gamma+\gamma \frac{(\gamma-1)}{2} M_{1}^{2}}$

On solving algebraically, we obtain the following result:

$\frac{P_{2}}{P_{1}}=\frac{\left(1+\gamma M_{1}^{2}\right)\left(\gamma M_{1}^{2}-\frac{(\gamma-1)}{2}\right)}{\left(1+\gamma M_{1}^{2}\right) \frac{(\gamma+1)}{2}}$

$\frac{P_{2}}{P_{1}}=\frac{2}{(\gamma+1)}\left(\gamma M_{1}^{2}-\frac{(\gamma-1)}{2}\right) \ldots \ldots$

Now applying equation 1 and 4 in:

$\frac{P_{\mathbf{m} 2}}{P_{1}}=\frac{P_{\mathbf{m} 2}}{P_{2}} * \frac{P_{2}}{P_{1}}$
$\frac{P_{\mathbf{m} 2}}{P_{1}}=\left[1+\frac{(\gamma-1)}{2} M_{2}^{2}\right]^{\frac{\gamma}{\gamma-1}} *\left[\frac{2 \gamma M_{1}^{2}-(\gamma-1)}{\gamma+1}\right]$

From equation 3 , we know $\mathrm{M}_{2}$ :

$\frac{P_{\mathbf{m} 2}}{P_{1}}=\left[1+\frac{(\gamma-1)}{2}\left(\frac{2+(\gamma-1) M_{1}^{2}}{2 \gamma M_{1}^{2}-(\gamma-1)}\right)\right]^{\frac{\gamma}{\gamma-1}} *\left[\frac{2 \gamma M_{1}^{2}-(\gamma-1)}{\gamma+1}\right]$

Let $\gamma$ for air $=1.4$, then our equation becomes:

$\frac{P_{\mathbf{m} 2}}{P_{1}}=\left[1+\frac{1}{5}\left(\frac{2+\frac{2}{5} M_{1}^{2}}{\frac{14}{5} M_{1}^{2}-\frac{2}{5}}\right)\right]^{\frac{7}{2}} *\left[\frac{\frac{14 M_{1}^{2}}{5}-\frac{2}{5}}{\frac{12}{5}}\right]$

$\frac{P_{\mathbf{m} 2}}{P_{1}}=\left[1+\frac{1}{5}\left(\frac{\frac{5+M_{1}^{2}}{5}}{\frac{7 M_{1}^{2}-1}{5}}\right)\right]^{\frac{7}{2}} *\left[\frac{7 M_{1}^{2}-1}{6}\right]$

$\frac{P_{\mathbf{m} 2}}{P_{1}}=\left[1+\frac{1}{5}\left(\frac{5+M_{1}^{2}}{7 M_{1}^{2}-1}\right)\right]^{\frac{7}{2}} *\left[\frac{7 M_{1}^{2}-1}{6}\right]$ 


$$
\begin{aligned}
& \frac{P_{\mathbf{m} 2}}{P_{1}}=\frac{\left(35 M_{1}^{2}-5+5+M_{1}^{2}\right)^{\frac{7}{2}}}{5^{\frac{7}{2}}\left(7 M_{1}^{2}-1\right)^{\frac{7}{2}}} *\left[\frac{7 M_{1}^{2}-1}{6}\right] \\
& \frac{P_{\mathbf{m} 2}}{P_{1}}=\left(\frac{36 M_{1}^{2}}{5}\right)^{\frac{7}{2}} * \frac{1}{\left(7 M_{1}^{2}-1\right)^{\frac{5}{2}}} * \frac{1}{6} \\
& \frac{P_{\mathbf{m} 2}}{P_{1}}=\frac{\left(\frac{6 M_{1}^{2}}{5}\right)^{\frac{7}{2}}}{\left(\frac{7 M_{1}^{2}-1}{6}\right)^{\frac{5}{2}}}
\end{aligned}
$$

Now we can easily know the value of $\mathrm{P}_{1}$, as it is the free stream static pressure behind the shock wave. For a given altitude, $\mathrm{P}_{1}$ can be known by the International Standard Atmosphere Chart.

Hence,

$$
\begin{aligned}
& P_{\mathbf{n} 2}=P_{1} \frac{\left(\frac{6 M_{1}^{2}}{5}\right)^{\frac{7}{2}}}{5} \ldots \ldots e q(5) \\
& \left(\frac{7 M_{1}^{2}-1}{6}\right)^{\frac{5}{2}}
\end{aligned}
$$

Now we know from equation 1:

$\frac{P_{\mathbf{m} 2}}{P_{2}}=\left[1+\frac{(\gamma-1)}{2} M_{2}^{2}\right]^{\frac{\gamma}{\gamma-1}}$

Applying equation 1 and substituting the value of $P_{\mathbf{m}_{2}}$ and $\mathrm{M}_{2}$, we get:

$$
P_{1} \frac{\left(\frac{6 M_{1}^{2}}{5}\right)^{\frac{7}{2}}}{\left(\frac{7 M_{1}^{2}-1}{6}\right)^{\frac{5}{2}}} * \frac{1}{P_{2}}=\left[1+\frac{(\gamma-1)}{2} * \frac{2+(\gamma-1) M_{1}^{2}}{2 \gamma M_{1}^{2}-(\gamma-1)}\right]^{\frac{\gamma}{\gamma-1}}
$$

Applying the value of $\gamma$ that is 1.4 for air:

$$
\begin{aligned}
& P_{1} \frac{\left(\frac{6 M_{1}^{2}}{5}\right)^{\frac{7}{2}}}{\left(\frac{7 M_{1}^{2}-1}{6}\right)^{\frac{5}{2}}} * \frac{1}{P_{2}}=\left[1+\frac{1}{5}\left(\frac{1+\frac{1}{5} M_{1}^{2}}{\frac{7}{5} M_{1}^{2}-\frac{1}{5}}\right)\right]^{\frac{7}{2}} \\
& P_{1} \frac{\left(\frac{6 M_{1}^{2}}{5}\right)^{\frac{7}{2}}}{\left(\frac{7 M_{1}^{2}-1}{6}\right)^{\frac{5}{2}}}=P_{2}\left[1+\frac{1}{5}\left(\frac{5+M_{1}^{2}}{7 M_{1}^{2}-1}\right)\right]^{\frac{7}{2}} \\
& P_{1} \frac{\left(\frac{6 M_{1}^{2}}{5}\right)^{\frac{7}{2}}}{\left(\frac{7 M_{1}^{2}-1}{6}\right)^{\frac{5}{2}}}=P_{2}\left[\frac{36 M_{1}^{2}}{35 M_{1}^{2}-5}\right]^{\frac{7}{2}}
\end{aligned}
$$




$$
P_{2}=P_{1} \frac{\left(\frac{6 M_{1}^{2}}{5}\right)^{\frac{7}{2}}}{\left(\frac{7 M_{1}^{2}-1}{6}\right)^{\frac{5}{2}}} *\left[\frac{35 M_{1}^{2}-5}{36 M_{1}^{2}}\right]^{\frac{7}{2}}
$$

Hence $\mathrm{P}_{2}$ is the pressure applied after the shock wave. Now assuming the area of the nose section of the aircraft to be conical, its area would be $\pi \mathrm{rl}$ (where $\mathrm{r}$ is the radius and 1 is the slant height)

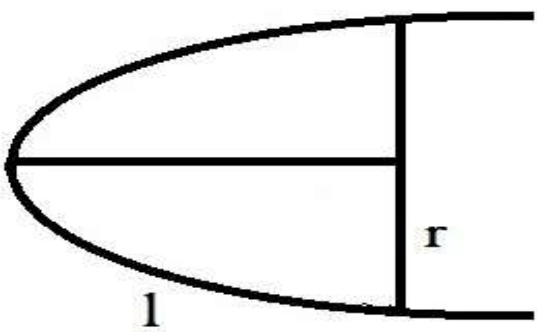

Figure 3 Nose section of the aircraft

The approximate area of this nose section would be:

$\mathrm{A}=\pi \mathrm{rl}$

And we know:

$F=P_{2} A$

$F=P_{1} \frac{\left(\frac{6 M_{1}^{2}}{5}\right)^{\frac{7}{2}}}{\left(\frac{7 M_{1}^{2}-1}{6}\right)^{\frac{5}{2}}} *\left[\frac{35 M_{1}^{2}-5}{36 M_{1}^{2}}\right]^{\frac{7}{2}} * \pi r l$

And hence this will be the force exerted after the shock wave on the surface of the nose. And this is the main result of our research, this equation can also be said as NBN's equation.

We can also prove the result of this research alternatively, by the famous Rayleigh's Pitot tube Formula:

Rayleigh's Pitot tube Formula:

$\frac{P_{\mathbf{m} 2}}{P_{1}}=\left[\frac{(\gamma-1)^{2} M_{1}^{2}}{4 \gamma M_{1}^{2}-2(\gamma-1)}\right]^{\frac{\gamma}{\gamma-1}} *\left[\frac{1-\gamma+2 \gamma M_{1}^{2}}{\gamma+1}\right]$

Now taking $\gamma=1.4$ (which is for air), the equation becomes:

$\frac{P_{\mathbf{m} 2}}{P_{1}}=\left[\frac{\left(\frac{12}{5}\right)^{2} M_{1}^{2}}{4\left(\frac{7}{5}\right) M_{1}^{2}-2\left(\frac{2}{5}\right)}\right]^{\frac{7}{2}} *\left[\frac{1-\frac{7}{5}+\frac{14}{5} M_{1}^{2}}{\frac{12}{5}}\right]$

$\frac{P_{\mathbf{m} 2}}{P_{1}}=\left[\frac{\frac{36}{25} M_{1}^{2}}{\frac{7 M_{1}^{2}-1}{5}}\right]^{\frac{7}{2}} *\left[\frac{14 M_{1}^{2}-2}{12}\right]$

$\frac{P_{\mathbf{m} 2}}{P_{1}}=\left[\frac{36 M_{1}^{2}}{5\left(7 M_{1}^{2}-1\right)}\right]^{\frac{7}{2}} *\left[\frac{7 M_{1}^{2}-1}{6}\right]$

$\frac{P_{\mathbf{m}}}{P_{1}}=\left[\frac{36 M_{1}^{2}}{5}\right]^{\frac{7}{2}} * \frac{1}{\left(7 M_{1}^{2}-1\right)^{\frac{5}{2}}} * \frac{1}{6}$ 


$$
\frac{P_{\mathbf{n} 2}}{P_{1}}=\frac{\left(\frac{6 M_{1}^{2}}{5}\right)^{\frac{7}{2}}}{\left(\frac{7 M_{1}^{2}-1}{6}\right)^{\frac{5}{2}}}
$$

This is nothing but equation 5 as proved earlier:

$$
\frac{P_{\mathbf{m} 2}}{P_{1}}=\frac{\left(\frac{6 M_{1}^{2}}{5}\right)^{\frac{7}{2}}}{\left(\frac{7 M_{1}^{2}-1}{6}\right)^{\frac{5}{2}}}=e q(5)
$$

From equation 5, we can easily get the value of $\mathrm{P}_{2}$ (pressure after the shock wave) and subsequently calculate force.

From equation 5, we can reach to the following result:

$P_{2}=P_{1} \frac{\left(\frac{6 M_{1}^{2}}{5}\right)^{\frac{7}{2}}}{\left(\frac{7 M_{1}^{2}-1}{6}\right)^{\frac{5}{2}}} *\left[\frac{35 M_{1}^{2}-5}{36 M_{1}^{2}}\right]^{\frac{7}{2}}$

And the force would be:

$$
F=P_{1} \frac{\left(\frac{6 M_{1}^{2}}{5}\right)^{\frac{7}{2}}}{\left(\frac{7 M_{1}^{2}-1}{6}\right)^{\frac{5}{2}}}\left[\frac{35 M_{1}^{2}-5}{36 M_{1}^{2}}\right]^{\frac{7}{2}} * \pi r l
$$

Hence from Rayleigh's Pitot tube Formula also we give our strong approach towards the research of pressure exerted on the surface after the shock wave. 